International Journal on Cloud Computing: Services and Architecture (IJCCSA),Vol.2, No.1,February 2012

\title{
"Secure Power Aware Routing to Support REAL TIME TRAFFIC IN MOBILE ADHOC NETWORKS"
}

\author{
Mrs. Sarita S. Gaikwad \\ Head of Dept., Department of Computer Engineering \\ ZES's Dnyanganga Polytechnic, \\ Narhe-41 Pune, Maharashtra \\ India \\ sarita.g1611@gmail.com
}

Prof. O. S. Rajankar

Principal., ZES’s Dnyanganga Polytechnic, Narhe-41 Pune, Maharashtra India

Prof. G.T Chavan

Asst. Professor,. Dept. Computer Enineering. Sinhgad College of Engineering,

Pune, Maharashtra India

gt. chavanegmail. com

Mrs. Shital M. Mate

Lecturer, Dept. of Computer Technology

Sou.Venutai Chavan Polytechnic

Pune, Maharashtra

India

sh.mmate@gmail.com

\begin{abstract}
:
A mobile Ad hoc network (MANET) is a collection of wireless nodes that forms a network without central administration. The nodes in such kind of network serve as routers as well as hosts. The nodes can forward packets on behalf of other nodes and run user applications. These devices are operated on battery which provides limited working capacity to the mobile nodes. Power failure and the energy consumption of the nodes is a critical factor in the operation of a mobile ad hoc network. The performance of the node can be hampered by power failure, which affects the ability of node to forward the packet and hence affects the
\end{abstract}

DOI : $10.5121 /$ ijccsa.2012.2104 
overall network lifetime. So an important objective is to consider Energy Aware design of network protocols for Ad hoc network environment. Different approaches can be applied to achieve the target and different energy-related metrics that have been used to determine energy efficient routing path. More efficient algorithm is proposed here, which tries to maximize the lifetime of network by minimizing the power consumption during the route establishment from source to destination. The proposed algorithm is incorporated with the route discovery phase of AODV and by simulation using NS/2 it is observed that the proposed algorithm is better than AODV in terms of packet delivery ratio and network lifetime.

\section{Keywords -}

MANET; AODV; EAR; DSR;

\section{INTRODUCTION}

Mobile ad-hoc network is a group of wireless mobile nodes that forms a provisional network without any centralized administration (Figure-2.1). In MANET the communication between the mobile nodes is done via multi-hop paths (Figure-2.2). It may be necessary for one node to enroll other nodes forwarding a packet to its destination due to the limited transmission range of wireless network interfaces[3]. Each mobile node operates as a host as well as a router forwarding packets for other mobile nodes in the network which may not be within the direct transmission range of each other. Each node participates in route discovery and in an ad-hoc routing protocol which allows it to determine multi-hop paths through the network to any other node. This idea of mobile ad-hoc network is also called infrastructure less networking[2], since the mobile nodes in the network dynamically establish routing among themselves to form their own network.

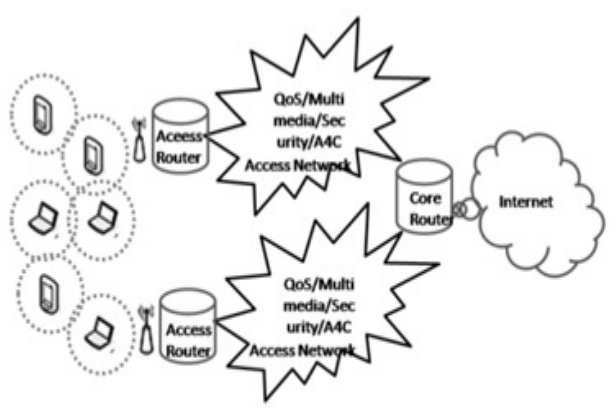

Figure A: Mobile Ad-hoc Network

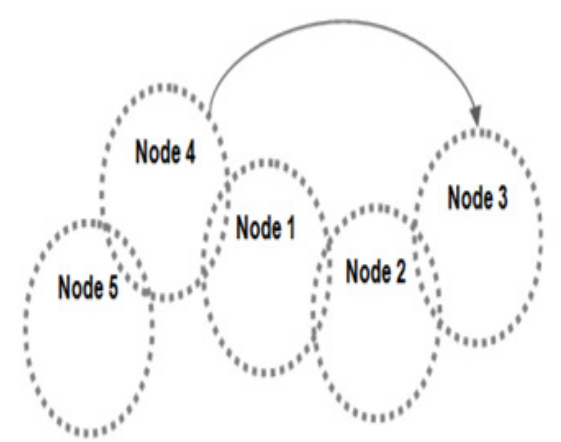

Figure B: Communication between nodes in MANET

Nodes operate in shared wireless medium. Network topology changes unpredictably and very dynamically. Radio link reliability is an issue. Connection breaks are very frequent. Furthermore, parameters like density of nodes, number of nodes and mobility of these hosts may vary in different applications. There is no stationary infrastructure.

A. MANET has the following features:

- Autonomous terminal: A node may function as both a host and a router.

- Distributed operation: No central administration therefore controls and management of the network is distributed among the terminals.

- Multihop routing: When delivering data packets from a source to its destination where no direct route is available. Then the packets should be forwarded via one or more intermediate nodes. 
- Dynamic network topology: As the nodes are mobile, the network topology may change quickly and not predictably and the connection between the mobile nodes may vary with time.

- Light-weight terminals: The mobile nodes in MANET have less CPU processing capability, small memory size, and low power storage. Therefore, these devices require optimized algorithms and mechanisms for computing and communicating functions.

\section{B. Following are applications of Mobile Ad Hoc:}

- Networks: The following are some well-known applications of MANET.

- Military: Automated battlefield, Special operations, Homeland defence

- Civilian: Disaster Recovery (flood, fire, earthquakes etc), Law enforcement (crowd control), Search and rescue in remote areas, Environment monitoring (sensors), Space/planet exploration

- Commercial: Sport events, festivals, conventions, Patient monitoring, Ad hoc collaborative computing (Bluetooth), Sensors on cars (car navigation safety), Vehicle to Vehicle communications, Video games at amusement parks, etc

\section{There are three types of routing protocols[1]:}

The main objective of any routing protocols is to reduce delay, increase network throughput, increase network lifetime and maximize energy efficiency. Due to dynamic change in topology, it is very difficult to maintain an optimal power aware route. Here, proposed method can be used to increase the network lifetime and reduce the power consumption during route establishment form source to destination. The proposed scheme is aimed to provide efficient real and non real time data transfer. Basically, following are the types of the routing protocols used in MANET:

1. Proactive Protocols: Destination sequenced distance vector (DSDV)

2. Reactive Protocols: Ad hoc on demand vector routing (AODV), dynamic source routing (DSR).

3. Hybrid Protocols

Ad hoc on demand vector routing: AODV $[2,3]$ is a method of routing messages between mobile computers. Unicast as well as multicast routing is possible with AODV. It allows these mobile nodes to communicate with the node which is not in range by passing messages through their neighbors. AODV performs this by finding the routes along which messages can be passed. AODV also checks that routes does not contain loops and finds the shortest route if possible. AODV has capability to handle dynamic changes in routes and can create new routes if there is an error in previous route. AODV builds routes by using route request (RREQ) / route reply (RREP) message cycles. When a source node desires a route to a destination for which it does not already have a route, it broadcasts a route request (RREQ) packet across the network. The nodes which receive this packet update information, and set up backwards pointers for source node in their route tables. A node receiving the RREQ can send a route reply (RREP) packet.

\section{EXISTING WORK}

Different approaches can be applied to achieve the target [2] Transmission power control and load distribution are two approaches which minimizes the active communication energy, and sleep/power-down mode is used to minimize energy during inactivity. The primary objective is to minimize energy consumption of individual node. The load distribution method tries to balance the energy requirement among the nodes and increases the network lifetime. This can be done by avoiding over-utilized nodes while selecting a routing path. Transmission power control approach, the stronger transmission power is used to increase the transmission range and reduces 
the hop count to the destination, if weaker transmission power is selected then it makes the topology sparse, which partitions the network and produces high end-to-end delay due to a larger hop count.

To determine energy efficient routing path, different energy-related metrics have been used like: Energy consumed/packet, Time to network partition, Variance in node power levels, Cost/packet, and Maximum node cost. Transmission power control approaches are discussed in Flow argumentation Routing (FAR)[3] where the network is considered as static network and tries to find the optimal routing path for a given source-destination pair that minimizes the sum of link costs along the path. Online Max-Min (OMM) [4] achieves the same but the data generation rate is not known in advance. Power aware Localized Routing (PLR) assumes that a source node has all location related information of its neighbors and the destination. Minimum Energy Routing (MER) [6] shows issues like obtaining accurate power information, associated overheads, maintenance of the minimum energy routes in the presence of mobility and implements the transmission power control mechanism in DSR and IEEE 802.11 MAC protocol. Some proposals considers load distribution approach are provides in Localized Energy Aware Routing (LEAR) Protocol [7] is based on DSR but modifies the route detection procedure for balanced energy consumption. In LEAR, a node concludes whether to forward the route-request message or not depending on its residual battery power. Conditional max-min battery capacity routing (CMMBCR) Protocol uses the concept of a threshold to exploit the lifetime of each node and to use the battery fairly.

\section{RELATED WORK ON POWER SAVING}

A lot of research is currently on going on to reduce the power consumed in each and every aspect of a mobile device. Power management is an significant technique to minimize the energy consumed in the wireless interface for mobile devices which are battery powered. The design of optimal power management policies needs to explicitly done for the diverse performance requirements posed by different application scenarios such as latency, throughput and other performance metrics.

Power management techniques have been studied extensively in the context of CPU, memory and disk management in the past. The main idea is to switch devices to the low-power state in periods of inactivity. As compared with traditional techniques in operating systems, power management in communication devices requires distributed coordination between two (or multiple) communicating entities, as all the entities have to be in the active mode for a successful communication.

When the arrival pattern of communication events is not known a priori, communication over the same wireless channel is required to inform a remote sleeping node to wake up for packets destined for it. This makes power management seemingly simpler. For example, if node A has packets destined for node B while node B is in the low power state, node A has to wait till node B becomes active before it transmits any packet. On the other hand, when node B is in the lowpower state, it has no idea that node A has packets destined for it.

Therefore, energy saving and performance inherently contradict each other in power managed wireless networks. A sophisticated design that minimizes the energy consumption may turn into the network non-operational. A power management policy in wireless networks is invoked to make the following decisions: 
International Journal on Cloud Computing: Services and Architecture (IJCCSA),Vol.2, No.1,February 2012

1. Which set of nodes should perform power management.

2. When a power-managed node switches to the low power state and

3. When a power-managed node switches from the low-power state to the active state.

A good power-saving topology management scheme for wireless ad hoc networks should have the following characteristics:

- Turn off radio receivers off because even an idle radio in receive mode can consume almost as much energy as an active transmitter.

- Forward data as well as control packet between source and destination with minimum delay.

- The algorithm should be distributed, requiring each node to make a local decision.

In wireless Ad hoc network power can be saved at different levels. Many researchers proposed different methods to reduce power consumption are as follows:

1. At Mobile device level

2. By controlling transmission level of packet

3. By using optimized power routing protocol

\section{At Mobile device level}

In [7] mobile devices power can be consumed in their sleep mode For example, like cell phones, as the trans-receiver is constantly hearing for signals, constant power drain is possible. Following are some techniques are given like

i. Disk scheduling

ii. CPU Scheduling

iii. Memory Allocation

So active node require more power as compared to sleep so if the node not participating in any work then this is best to send that node in sleep mode which saves power consumption of that node. The network hardware at receiver node can operate in any four different modes In [9] author present the algorithms for sleep mode scheduling i.e. Adaptive sleep algorithm and Birthday sleep algorithm. This algorithm consider all of the nodes of ad hoc network not always participate in receiving, sending and forwarding of data packet, rather many nodes do not need to be logically in the topology to maintain connectivity. If these nodes can be identified then they can be put into sleep mode rather then staying in ideal mode which results in power saving at that node

\section{By controlling transmit power level}

Selecting the transmit power for each packet in a distributed fashion at each node is the power control problem in wireless ad hoc networks. This is the complex problem as the choice of the power level basically affects many features of the operation of the network:

i. Quality of signal received can be determined from the transmit power level.

ii. It determines the range of a transmission.

iii. It determines the magnitude of the interference it creates for the other receivers.

Because of these factors:

i. Due to (i) physical layer is affected. 
International Journal on Cloud Computing: Services and Architecture (IJCCSA),Vol.2, No.1,February 2012

ii. Network layer is affected since the transmission range affects routing (due to ii).

iii. Transport layer is affected because interference causes congestion (due to iii).

Transmit power control is thus a ideal cross layer design problem affecting all layers of the protocol stack from physical to transport. Therefore the cross-layer design should be used with some concern. Question arise here like where in the network architecture power control should be located.

\section{B. Design principles for power control protocol}

Following are the design principles for power control:

- To maximize network capacity it is most favourable to reduce the transmit power level.

- $\quad$ Reducing the transmit power level reduces the average contention at the MAC layer.

- The impact of power control on total energy consumption depends on the energy consumption pattern of the hardware.

- When the traffic load in the network is high, a lower power level gives higher end-to-end delay, where when the traffic load in the network is low, a higher power gives lower delay.

- $\quad$ Power control can be regarded as a network layer problem.

\section{By using optimized power aware routing protocol}

In wireless ad hoc networks, every host acts both as a router and a packet sender, so the classical routing protocols used by wired networks are not applicable at all to MANETs. Existing routing protocols may be classified following three criteria:

- Based on the logical organization through which the protocol "describes" the network

- Based on the way routing information is obtained

-

From this point of view, protocols may be divided in

1. Reactive (or On-Demand)

2. Proactive

3. Hybrid.

- Based on how the routing path is created: From this point of view, protocols may split in two

i. Source Routing

ii. Non Source Routing.

\section{NEED FOR POWER AWARE ROUTING (PAR)}

A. Problem analysis : A mobile Ad hoc network (MANET) is a group of mobile wireless nodes that forms a network which is independent of any centralized administration during the packet forwarding to each other in multiple-hop fashion. As those devices are operated on battery, the energy consumption of the nodes is a critical factor in the operation of a mobile adhoc network. So it is an important objective that motivates the researches and practitioners to consider Power Aware design of network protocols for ad hoc network environment. 
International Journal on Cloud Computing: Services and Architecture (IJCCSA),Vol.2, No.1,February 2012

Routing in MANET is challenging due to node mobility, limitations for transmission bandwidth, battery power, and CPU time. In MANET nodes cooperate in routing the packets to destination. Each node in the network communicates only with those nodes that are located within its communication range. The distance between source and destination may be at multiple hopes. Death of few or even single node due to energy exhaust will cause the breakdown in communication of entire network. While taking accumulated energy we will check the status of each node can be estimated after transmitting the required level path will be discarded.

Also as the type and size of data known, the battery status of every node can be estimated after transmitting the required data, care will be taken while selecting the route such that any node does not get exhausted completely after the data transmission and there by become dead. In such case the alternate route will be selected.

The estimation of battery status can be done from the details send by the node when it sends route request packet. In route request packet the header file has the following information. Source_id, Destination_id, Type of Data to be transfer, Total Battery Status, Total Traffic level and Node_id. Total traffic level is calculated from the packets buffered in the interface queue of the node.

B. Objective: The energy of nodes is battery powered with limited energy tank. It is not convenient to recharge or replace the batteries of the nodes hence energy preservation is crucial. An energy efficient routing protocol balances node energy exploitation to minimize energy consumption and maximize the life of nodes. Thus, the network lifetime cane be maximized, routing delay can be minimized and increasing the reliability of the packets reaching the destination. MANET does not have any fixed communication infrastructure. For any active connection the source, destination as well as the intermediate nodes can change their position due to the mobility nature. Therefore routes are subject to frequent disconnection. In such situation it is vital to decrease disruptions caused by changing topology.

Energy Efficient Power Aware Routing protocol considerably decreases the total number of route request packets(RREQ), which results in an increased packet delivery ratio, minimizing end- to-end delays for the data packets, lower control overhead, fewer collisions of data and control packets, supporting consistency and minimizing power consumption. Each route request carries the cumulative cost, so very little bit overhead is increased to carry the cumulative cost but it is negligible. Implementation of path selection is based on energy threshold of individual node and a node energy after transmitting known data volume.

\section{SECURE POWER AWARE ROUTING: SPAR}

The proposed algorithm increases lifetime of network and reduces the power expenditure during the route establishment using a secure cryptographic method. Only the secure node having required energy level can participate in route discovery phase and data transmission. This algorithm can transfer both real time and non real traffic by providing energy efficient and less congested path between a source and destination. Algorithm focuses on basic 3 parameters[1]: 
International Journal on Cloud Computing: Services and Architecture (IJCCSA),Vol.2, No.1,February 2012

1) Accumulated Energy of a path

$$
E_{i j}=\sum_{i=1}^{j-1} E_{i}
$$

$E_{i}$ is the residual energy of an intermediate node $i$ and $E_{i j}$ is the total energy of a path from node $i$ to node $j$.

2) Status of Battery Lifetime $\left(B \_S\right)$

3) Type of Data to be transfer:

a. Non Real Time (NRT)

b. Real time $(R T)$.

\section{A. Parameters on each node}

\section{Each node has 3 variables:}

1. Node_ID : Used for node identification. Each node is identified by unique ID.

2. $\quad$ Battery Status $\left(B \_S\right)$ : Total energy at node.

3. Traffic Level $\left(T_{-} L\right)$ : Number of packets stored in the interface queue of the node.

\section{Battery status is further divided into 3 categories:}

4. If (Battery Status $<20 \%$ )

Then Set $B \_S=1$

5. If $(20 \% \leq \square$ Battery Status $\leq \square 60 \%)$

$$
\text { Then Set } B \_S=2
$$

6. If (Battery Status $\geq \square 60 \%$ )

Then Set $B \_S=3$

\section{B. Parameters to concern during route search}

At the time of route discovery phase, a route request (RREQ) packet send or broadcasted by the source to all its neighbor nodes for getting information about destination. RREQ packet's header includes source_id, destination_id, $T \_O \_L$ (type of data to be transfer), $T \_B \_S$ (Total Battery Status), $T \_T \_L$ (Total Traffic Level), and Node_IDs.

\section{Calculation of total battery status (t_b_s)}

At source node $T \_B \_S=0$. As RREQ packet propagate along the path, $T \_B \_S$ is updated at each intermediate node $i$ as follows:

$$
\begin{aligned}
& \text { If }\left(B \_S i==3\right) \\
& \text { Then } T \_B \_S=T \_B \_S+3 \\
& \text { Else-if }\left(B \_S i==2\right) \\
& \text { Then } T \_B \_S=T \_B \_S+1 \\
& \text { Else-if }\left(B \_S i==1\right)
\end{aligned}
$$

No updation is performed, and the node is not allowed to participate in the route discovery.

\section{Calculation of total traffic level $\left(\mathbf{t} \_t \_l\right)$}

1. Initially, at source node, $T \_T \_L=0$.

2. Add traffic status of each intermediate node to $T_{-} T_{-} L$ during route discovery.

Traffic level (T_L) is number of packets stored in the interface queue of the node. 


\section{E. Route selection criteria at destination side}

The destination waits for a threshold time $\left(T_{t h}\right)$ after a RREQ packet arrives. Destination determines the link status ratio(L_S_R $)$ of the route for every arrived RREQ packet[1]. Destination stores all possible route request for a certain amount of time (Threshold time). After expiry of timer, the destination node selects the route with the required L_S_R and replies for a path accordingly with secured node. Here link status ratio of a path is calculated using equation (2):

$$
R=\frac{E_{i j}}{H_{n}}
$$

Where $E_{i j}$ is the 1 energy of path from node $\mathrm{i}$ to node $\mathrm{j}$ (equation 1 ). $H_{n}$ is number of intermediate hops along the path.

\section{F. Energy consumption model}

Energy consumption of a node after time t is calculated using equation (3):

$$
E_{c(t)}=N_{t} * \alpha+N_{r} * \beta
$$

Where $\boldsymbol{E} \boldsymbol{c}(\boldsymbol{t})$, energy consumed by a node after time t.

$N \boldsymbol{t}$ - Number of packets transmitted by the node after time t.

$\boldsymbol{N} \boldsymbol{r}$ - Number of packets received by the node after time $\mathrm{t}$. $\alpha$ and $\beta$ are constant factors having a value between $O$ and 1 .

If $E$ is the initial energy of a node, the remaining energy $\operatorname{Er}(t)$ of a node at time t, is calculated using equation (4):

\section{G. Algorithm: spar}

$$
E_{r(t)}=\square E-\square E_{c(t)}
$$

$$
\text { If }\left(T \_O \_L==N R T\right)
$$

Let $\mathrm{N}$ different values of $\mathrm{R}$ are received, Where $R \geq 1$

$$
\text { if }(N=0)
$$

Send negative acknowledgement to the source that path cannot be established.

$$
\text { else-if }(N==1)
$$

Acknowledge the source with this path.

$$
\text { else-if }(N>1)
$$

Select the path with $\min \left\{T_{-} T \_L\right\}$

Acknowledge the source with the selected path.

$$
\text { else-if }\left(T \_O \_L==R T\right)
$$

Let $\mathrm{N}$ different values of $\mathrm{R}$ are received, where $R \geq \square 2$ 
International Journal on Cloud Computing: Services and Architecture (IJCCSA),Vol.2, No.1,February 2012

$$
\text { If }(N==0)
$$

Send negative acknowledgement informing that no such path is possible.

$$
\text { else-if }(N==1)
$$

Acknowledge the source with this path.

$$
\text { else-if }(N>1)
$$

Select the path with Min $\left\{T_{-} T_{-} L\right\}$

Acknowledge the source with the selected path.

\section{SIMULATION AND RESULTS}

- The proposed scheme is simulated using network simulator NS-2[9] with latest version NS-2.35 and the performance is compared with well known protocol AODV and the Power aware routing (PAR).

- Scenarios have been setup for 20 , and 50 nodes in an area of $1000 \mathrm{~m} * 1000 \mathrm{~m}$. In the different scenarios from small network to large networks, value for packet delivery ratio has been observed by varying pause times from 0 to 500 and the speed has been changed form 1 meter per second to 25 meters per second.

- Initially scenario has been setup for a 20 nodes network as shown in figure 1(a) and figure 2(a). As shown in figure 1(a) speed is constant and pause time is varied. In the beginning of the simulation, performance of AODV dips slightly, the reason can be delay in route reply messages due to high mobility of nodes and then once AODV stabilizes it is delivering more packets. PAR scheme performs better as number of nodes increases. There is a slight delay in the start as it takes time to calculate the values for different parameters like power status, Traffic level, number of hops etc. Once initial calculations are done, PAR is able to deliver more than $97 \%$ packets all the time and at pause time greater than 250 it delivers approximately $99 \%$ packets. SPAR shows the similar results as compared to PAR. While in case of figure 2(a) pause time is kept constant i.e. 10 seconds and speed is varied. In this case also SPAR is delivering more packets as compare to AODV for all speeds from $0 \mathrm{~m} / \mathrm{sec}$ to $20 \mathrm{~m} / \mathrm{sec}$.

- Now Scenario has been setup for 50 nodes. The dense medium changes some features of the protocols under study. As shown in figure 1(b) the performance of proposed algorithm 'SPAR' is best for 50 nodes proving the point that it was better to take care of factors like energy status and traffic level. Although initially packet fraction was very less when pause time was zero but later on as pause time increased performance of SPAR in much better as compare to AODV in terms of packet delivery. In figure 2(b), again speed is varied and pause time is kept constant that is again 10 seconds. It can be seen from the graph that again SPAR is much better than its counterpart AODV and PAR for 50 nodes (dense medium). SPAR is delivering more number of packets for all speeds from $0 \mathrm{~m} / \mathrm{sec}$ to 20 $\mathrm{m} / \mathrm{sec}$. The reason is again selection of a better path having less no. of hops, better energy status and minimum traffic level 
- SPAR uses IP level HELLO messaging to detect link breakages. If HELLO is not received within one second, the link is assumed to be broken. An active route timeout is after 50 seconds if unused. The reverse route lives less that is only 10 seconds. The route reply message should be received within one second after the request. If any of the nodes does not answer HELLO once, it is assumed that the link is broken. Route discovery is only tried three times. Request retransmits are done with three seconds intervals. Packets are held eight seconds while they are awaiting their routes to be discovered. Again, a node can send one route reply at each second. Energy status is attached with each HELLO packet; it is decremented by factor 0.025 each time a HELLO is echoed. Power status is attached with each HELLO packet; it is decremented by factor 0.025 each time a HELLO is echoed. Power status has been set at a scale of 7-10 at start for all nodes to be in active state. The entry is made in the route table at reply message stage. In case of proposed scheme route tables are updated at each Hello interval as in AODV with added entries for energy status and other factors.

- Performance comparison of 'SPAR' with AODV and PAR in all above discussed scenarios is shown graphically in figure 1 , figure 2 , and figure 3 . A special random scene has been considered in Figure 3, in this scenario all nodes are configured with different pause times and different speed and packet delivery ratio is observed by varying number of nodes as 10,20 and 50. Results show that the proposed scheme outperforms as the network grows and become larger and more dynamic. Even in case of a complete random scene the performance of SPAR is better than simple AODV and PAR as number of nodes increased in the network as shown in Figure 3.

- At last an experiment for a network of 20 nodes is performed for network lifetime. Network lifetime has been considered as the time in which a percentage of nodes are completely exhausted and the network is considered as a dead network. Simulation study is done for different speeds with constant pause time of $10 \mathrm{~ms}$. Figure 4(a) and Figure 4(b) shows the comparison of network lifetime between simple AODV, PAR and SPAR with a speed of $2 \mathrm{~m} / \mathrm{s}$ and $5 \mathrm{~m} / \mathrm{s}$ respectively. It can be easily observed by Figure 4(a) that at a speed of $2 \mathrm{~m} / \mathrm{s}$, the network life time of SPAR is increased by $22.2 \%$ as compare to simple AODV therefore lifetime of proposed algorithm is increased with a good factor. While in Figure 4(b), it is clear that the network lifetime of SPAR is increased by $15.4 \%$ as compare to simple AODV. Although speed of $5 \mathrm{~m} / \mathrm{sec}$ is fairly high in terms of energy consumption in a dynamic network but still SPAR is maintaining more network lifetime as compare to simple AODV.
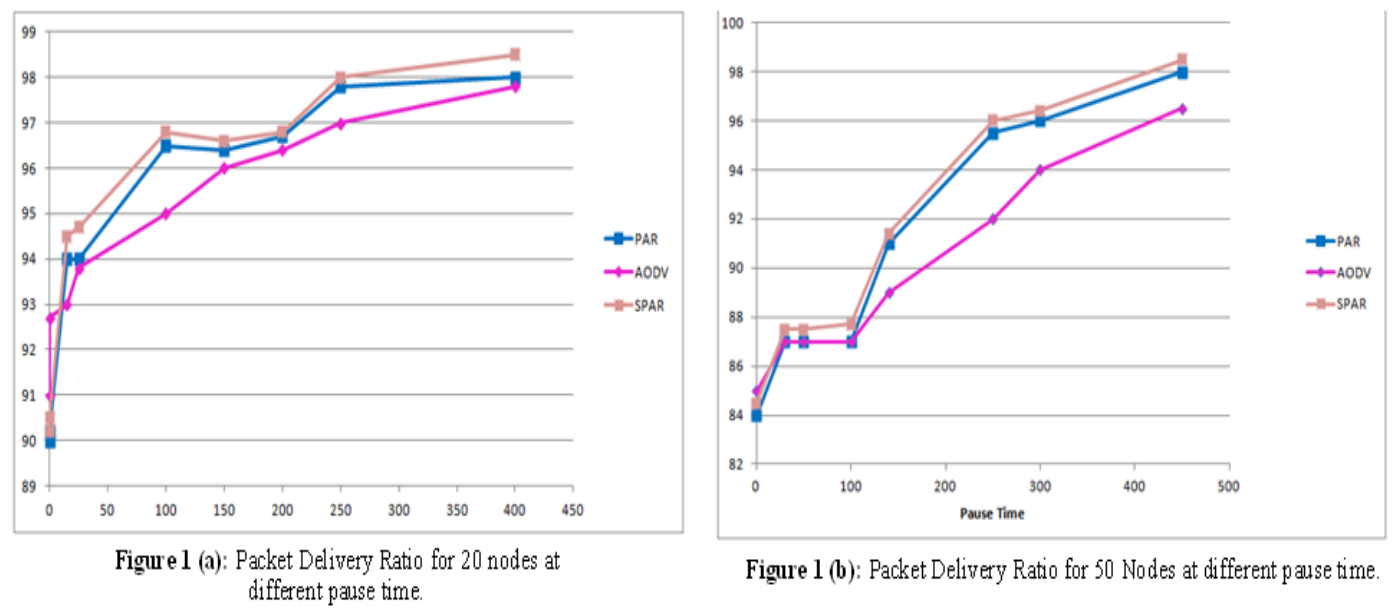

Figure 1(b): Packet Delivery Ratio for 50 Nodes at different pause time 


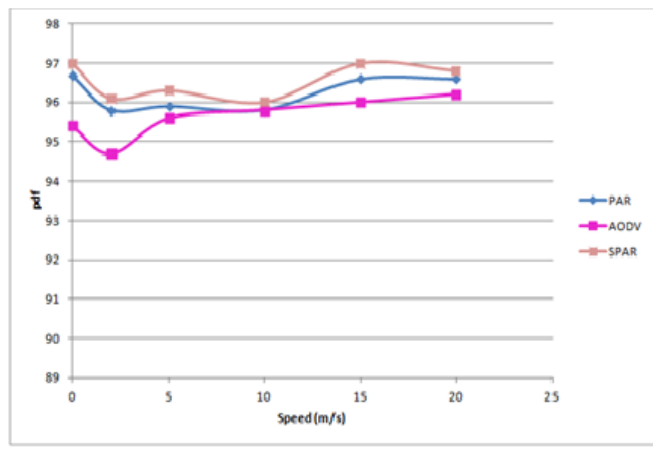

Figure 2(a): Packet Delivery Ratio for 20 nodes ? $^{+}$ different speed.

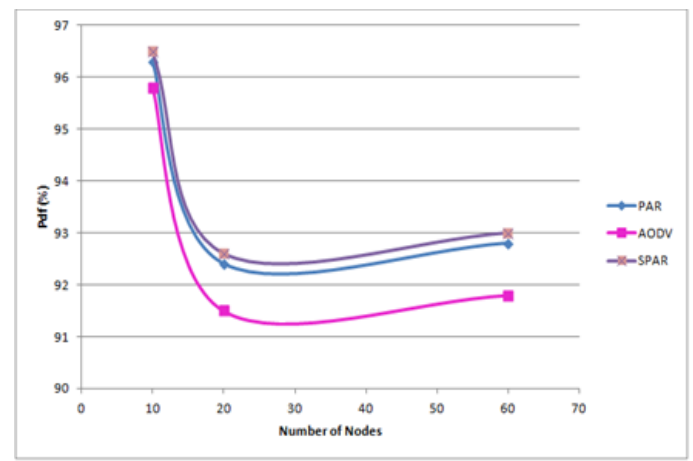

Figure 3: Packet Delivery Ratio for Random Scen

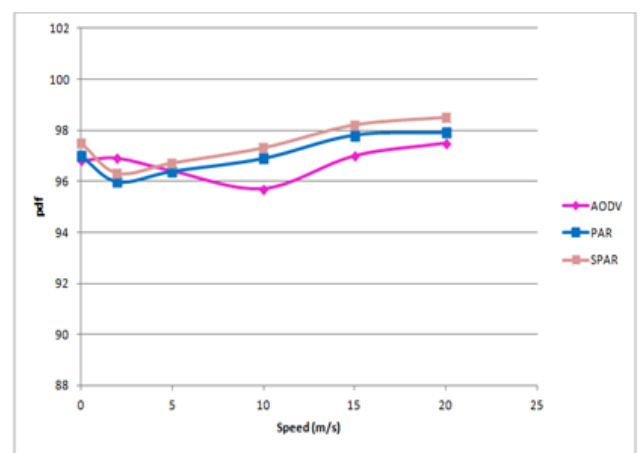

Figure 2(b): Packet Delivery Ratio for 50 nodes at different speed.

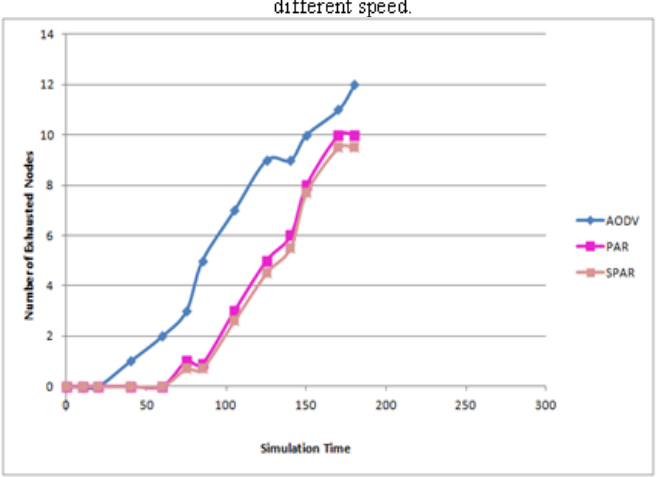

Figure 4(a): Simulation time vs. exhausted nodes with a speed of $2 \mathrm{~m} / \mathrm{s}$.

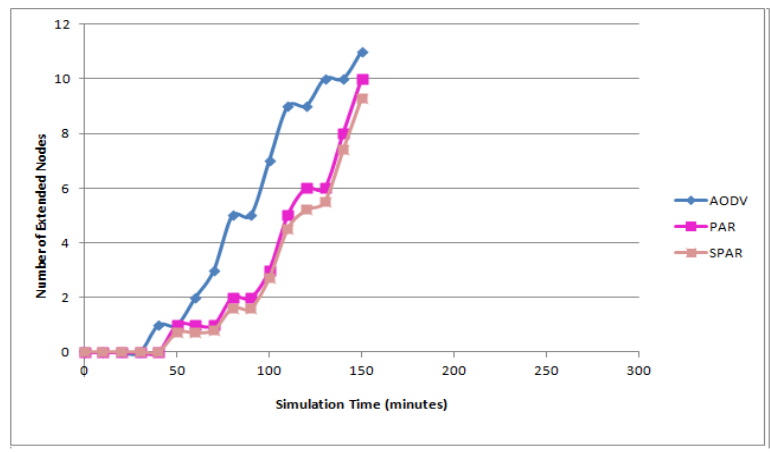

Figure 4(b): Simulation Time vs. Exhausted nodes with a speed of $5 \mathrm{~m} / \mathrm{s}$.

\section{CONCLUSION}

Energy efficiency is one of the main problems in a mobile ad hoc network, especially designing a routing protocol. The proposed work aims at discovering an efficient power aware routing scheme in MANETs and analyzing the derived algorithm with the help of NS-2. Simulation result shows that the proposed scheme SPAR as well as PAR is delivering more packets in different network scenarios as well as network life time of the PAR and SPAR is better even in high mobility scenarios. 
The process of checking the proposed scheme is on for more sparse mediums and real life scenarios and also for other metrics like Path optimality, Link layer overhead, total energy consumed etc. Although this scheme can somewhat enhance the latency of the data transfer but it results in a significant power saving and long lasting routes. This scheme is one of its types in adhoc networks which can provide different routes for different type of data transfer and ultimately increases the network lifetime.

\section{REFERENCE}

[1] Ajina A , G.R.Sakthidharan, Kanchan M. Miskin "Study of Energy Efficient Power Aware Routing Algorithm and their Applications" This paper appears in: 2010 Second International Conference on Machine Learning and Computing 978-0-7695-3977-5/10 \$26.00 (C) 2010 IEEE DOI 10.1109/ICMLC.2010.44

[2] AL-Gabri Malek, Chunlin Li, Li Layuan, WangBo "New Energy Model: Prolonging the Lifetime of Ad-hoc On-Demand Distance Vector Routing Protocols (AODV)" "2010 International Conference 978-1-4244-5824-0/\$26.00_c 2010 IEEE

[3] Alokes Chattopadhyay, Markose Thomas, Arobinda Gupta "An Energy Aware Routing Protocol for Mobile Ad-Hoc Networks" 15th International Conference on Advanced Computing and Communications 0-7695-3059-1/07 \$25.00 ( ) 2007 IEEE DOI 10.1109/ADCOM.2007.70

[4] Forman G., Zahorjan J., “The Challenges of Mobile Computing,” IEEE Computer 1994; 27(4):38-47.

[5] Morteza Maleki, Karthik Dantu, and Massoud Pedram "Power-aware Source Routing Protocol for Mobile Ad Hoc Networks" This research was sponsored in part by DARPA PAC/C program under contract no. DAAB07-00-C-L516. ISLPED’02, August 12-14, 2002, Monterey, California, USA.

[6] Perkins C., "Ad Hoc Networking” Addison-Wesley: 2001; 1-28.

[7] Pinyi Ren, Jia Feng and Ping Hu "Energy Saving Ad-hoc On-Demand Distance Vector Routing for Mobile Ad-hoc Networks" This full text paper was peer reviewed at the direction of IEEE Communications Society subject matter experts for publication in the IEEE ICC 2009 proceedings 978-1-4244-3435-0/09/\$25.00 @2009 IEEE

[8] Qu Lei Wang Xiaoqing “ Improved Energy Aware AODV Routing Protocol” This paper appears in: 2009 WASE International Conference on Information Engineering 978-0-7695-3679-8/09 @2009 IEEE. DOI 10.1109/ICIE.2009.96

[9] Rachit Jain, Naresh B. Khairnar, Laxmi Shrivastava "Comparative Study of Three Mobile Ad-hoc Network Routing Protocols under Different Traffic Source "2011 International Conference on Communication Systems and Network Technologies 978-0-7695-4437-3/11 \$26.00 (C) 2011 IEEE DOI 10.1109/CSNT.2011.28

[10] Rahul C. Shah and Jan M. Rabaey "Energy Aware Routing for Low Energy Ad Hoc Sensor Networks" This research was supported by DARPA on grant no. F29601-99-1-0169 entitled, "Communication/Computation Piconodes for Sensor Networks"

[11] Rakesh K. Kadu, Narendra V. Chaudhari "A Study of Power Saving Techniques in Mobile Adhoc Network" This paper appears in:Internation Conference on Computer Science and Information Technology 2008. 978-0-7695-3308-7/08 @2008 IEEE. DOI 10.1109/ICCSIT.2008.142

[12] Rizvi, S.A.M.; Hussain, S.Z.; Wadhwa, N "Performance Analysis of AES and TwoFish Encryption Schemes" this paper appears in: Communication Systems and Network Technologies (CSNT), 2011 International Conference Issue Date: 3-5 June 2011 On page(s): 76 - 79 E-ISBN: 978-0-7695-4437-3

[13] Shilpa Banerjee, Piu Bera, S. Choudhury, Moitreyee Dasgupta "A Power Aware Multicast Ondemand Routing with Load Balancing “ This paper appears in 2010 2nd International Conference on Computer Technology and Development (ICCTD 2010)

[14] Suresh Singh and Mike Woo, C.S.Raghavendra "Power Aware Routing in Mobile Adhoc Network" This paper appears in: MOBICOM 98 Dallas Texaas USA . ACM 1-58113-035-x/98/10

[15] Vinay Rishiwal, Mano Yadav and Shekhar Verma "Power Aware Routing to Support Real Time Traffic in Mobile Ad hoc Networks" This paper appears in: International Conference On "Emerging Trades in Engineering and Technology" 978-0-7695-3267-7/08, DOI 10.11.09/ICETET.2009.108

[16] The ns Manual The VINT Project November 4, 2011 